\title{
Bladder carcinosarcoma: A case report with review of the literature
}

\author{
Ismail Basibuyuk $^{1}$, Ramazan Topaktaş ${ }^{2}$, Fatih Elbir $^{3}$ \\ ${ }^{1}$ Cizre State Hospital, Department of Urology, Sirnak, Turkey; \\ ${ }^{2}$ Haydarpasa Numune Training and Research Hospital, Department of Urology, Istanbul, Turkey; \\ ${ }^{3}$ Mardin State Hospital, Department of Urology, Mardin, Turkey.
}

\begin{abstract}
Summary Carcinosarcoma of the urinary bladder is a rare neoplasm that is histologically composed of malignant epithelial and mesenchymal components. The etiology of sarcomatoid tumors is unclear, but smoking and history of previous radiotherapy or chemotherapy may lead to bladder disorders and to the formation of sarcomatoid carcinoma. These neoplasms behave as highly aggressive tumors and optimal treatment is uncertain. Herein, we report a case of sarcomatoid carcinoma of urinary bladder presenting as a giant intravesical mass in a 61-year-old man complaining of macroscopic hematuria.
\end{abstract}

KEY WORDS: Bladder carcinosarcoma; Urothelial carcinoma; Prognosis.

Submitted 11 June 2017; Accepted 19 July 2017

\section{INTRODUCTION}

The majority of bladder cancers (95 to 98\%) originate from the urothelium (1). Carcinosarcomas or sarcomatoid carcinomas are extremely rare malignancies, which have a biphasic character involving epithelial and mesenchymal components (2). Loss of heterozygosity in stem cells is considered as the main factor in the underlying development of sarcomatoid carcinomas $(2,3)$. In addition, cyclophosphamide chemotherapy, smoking, and radiotherapy are considered to play a role in the etiology $(4,5)$. In most cases, epithelial component includes high gradeurothelial carcinoma, whereas mesenchymal component includes a chondrosarcoma, malignant fibrous histiocytoma, osteosarcoma, leiomyosarcoma, and rhabdomyosarcoma (5-7). Herein, we present a paraplegic case who was diagnosed with a bladder carcinosarcoma in the light of literature data.

\section{Case report}

A 61-year old, paraplegic male patient was admitted to our clinic with painless, gross hematuria with clots for three days. He had a history of coronary artery bypass grafting 12 years ago, spinal anesthesia-related paraplegia which developed two years before, and congestive heart failure (ejection fraction: $35 \%$ ). He was a smoker. On examination, the paraplegic patient

Figure 1. within the bladder. had a catheter without any other remarkable finding. Laboratory test results were normal. Whole abdominal ultrasonography (USG) showed a 9 × $8 \mathrm{~cm}$ mass which filled the bladder. Abdominopelvic computerized tomography (CT) showed a $9 \times 7 \mathrm{~cm}$ mass lesion, originating from the right lateral wall of the bladder and occupying the entire bladder (Figure 1). A written informed consent was obtained from the patient and cystoscopic examination was performed under general anesthesia. The mass, which originated from the bladder neck and filled the bladder, was incompletely resected. Pathological examination showed a biphasic pattern, and the result was reported as a sarcomatoid carcinoma. The epithelial component included an adenocarcinoma and squamous-cell carcinoma, whereas the sarcomatous component included a spindle-cell and chondrosarcoma. As the all resection specimen consisted of tumor tissues, we were unable to evaluate the depth of invasion.

Computed tomography showed no sign of lymph node or organ metastasis. Four weeks after transurethral resection (TUR-BT), radical cystoprostatectomy, lymph node dissection, and ileal conduit surgery were performed. Cystoprostatectomy specimen had a $10 \times 8.5 \mathrm{~cm}$ tumor in-diameter (Figure 2). After histological examination, tumor was reported as a high-grade sarcomatoid carcinoma, pT2a, pNO, pMx. The histological pattern consisted of $70 \%$ sarcomatous component (spindle-cell and chondrosarcoma), and 30\% epithelial component (adenocarcinoma and squamous-cell carcinoma). All surgical margin samples and lymph nodes were reported as normal. Four days after the surgery, the patient died due to myocardial infarction.

A computed tomography image of a $9 \times 7 \mathrm{~cm}$ heterogeneous mass

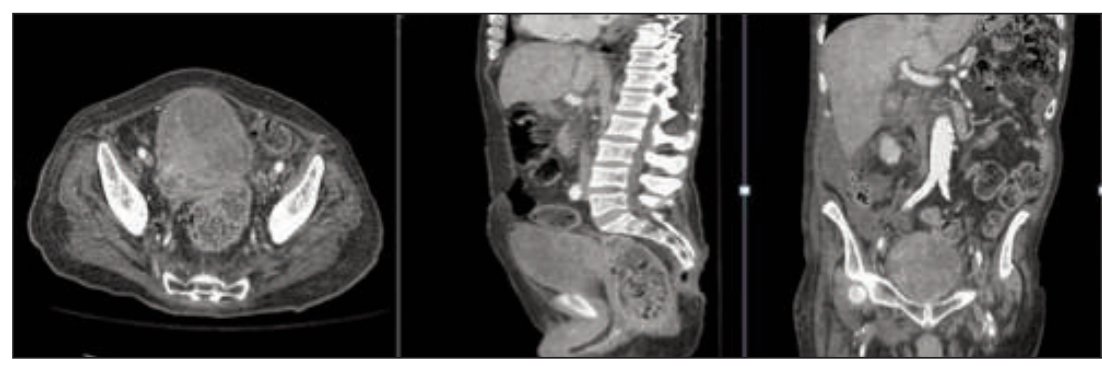

No conflict of interest declared. 
Figure 2.

A gross view of a solid tumor invading the bladder completely.

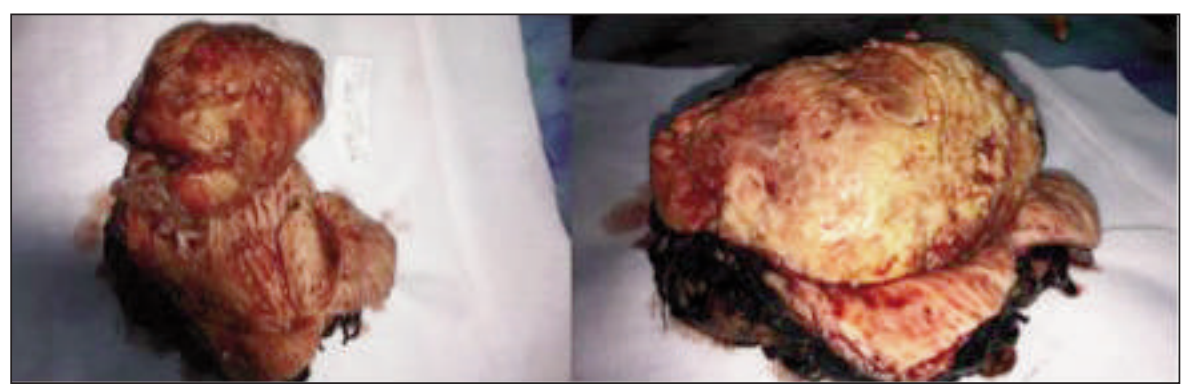

incomplete resection (8). Recently, the combination of radical cystoprostatectomy and lymphadenoctomy with neoadjuvant or adjuvant chemotherapy and/or radiotherapy has been recommended (11).

On the other hand, cancer-specific survival of carcinosarcomas is extremely poor, and tumor grade and subtype of epithelial component are the most important factors affecting survival $(11,12)$.

\section{Discussion}

Bladder carcinomas are extremely rare tumors, which show polypoid character, and can reach large sizes, as assessed in gross examination. Similar to other types of bladder cancer, these tumors are more common in males, and the incidence increases with age (8).

Tumors usually originate from the lateral walls, dome, trigon, and anterior wall (8). Smoking, cyclophosphamide, and radiotherapy are considered to play a role in the etiology of carcinosarcomas due to their undesired effects on the cell proliferation $(2,4)$.

Similar to all other bladder cancers, these patients are admitted with painless gross hematuria. Dysuria, increased need to urinate, and obstructive symptoms can be also seen (9).

In our case, the patient was admitted with painless gross hematuria and a $10 \mathrm{~cm}$ mass originated from the right lateral wall of the bladder.

In most cases, the epithelial component is reported as a high-grade papillary urothelial carcinoma $(5,6)$.

Sarcomatous component, on the other hand, usually include one or more of the followings: chondrosarcoma, leiomyosarcoma, and malignant fibrous histiocytoma (5-7). In our case, pathological examination showed that the sarcomatous component consisted of a spindle-cell and chondrosarcoma, whereas the epithelial component consisted of an adenocarcinoma and squamous-cell carcinoma. Furthermore, sarcomatoid tumors may affect several organs; however, clinical progression may vary according to the site (10).

The best prognosis is seen, when the tumors involve the respiratory and gastrointestinal system, whereas tumors located in kidneys, bladder, prostate, and stomach have a poor prognosis (10). Bladder carcinosarcomas are considerably aggressive, and there is no consensus on the standard treatment of these malignancies. However, TUR-BT, radical cystectomy, radical cystectomy + radiotherapy, partial cystectomy + neoadjuvant radiotherapy followed by radical cystectomy are used for the treatment. Although the efficacy of these methods is controversial, the main treatment methods are radical cystectomy and lymph node dissection $(8,11)$.

Aggressive surgery during early period is the only curative treatment option (5).

In addition, radical cystectomy can be performed effectively in patients who have superficial or deep invasion, whereas transurethral resection and partial cystectomy are usually not preferred due to the increased risk of
While the majority of patients die within the first year, some patients live longer than 10 years (10). According to a recent study, one-, five-, and 10 years survival rates for bladder carcinosarcomas are 53.9\%, 28.4\%, and $25.8 \%$, respectively (10).

Unfortunately, our case died due to myocardial infarction four days after radical cystoprostatectomy.

\section{Conclusions}

Due to aggressive and fatal nature of carcinosarcomas, a radical treatment approach should be implemented immediately as possible after the diagnosis.

Considering available data on carcinosarcomas in case reports, further large-scale studies should be carried out to gain a better understanding of the biological basis of this disease and to develop targeted therapies.

\section{References}

1. Erdemir F, Uluocak N, Tunc M, et al. Sarkomatoid carcinoma of the urinary bladder. Turk J Urology. 2006; 32:462-6.

2. Mukhopadhyay S, Shrimpton AE, Jones LA, et al. Carcinosarcoma of the urinary bladder following cyclophosphamide therapy: evidence for monoclonal origin and chromosome 9p allelic loss. Arch Pathol Lab. 2004; 128:8-11.

3. Halachmi S, DeMarzo AM, Chow NH, et al. Genetic alterations in urinary bladder carcinosarcoma: evidence of a common clonal origin. Eur Urol. 2000; 37:350-7.

4. Maestroni U, Giollo A, Barbieri A, et al. Bladder carcinosarcoma: a case observation. Acta Biomed. 2004; 75:74-6.

5. Lopez-Beltran A, Pacelli A, Rothenberg HJ, et al. Carcinosarcoma and sarcomatoid carcinoma of the bladder: clinicopathological study of 41 cases. J Urol. 1998; 159:1497-503.

6. Lahoti C, Schinella R, Rangwala AF, et al. Carcinosarcoma of urinary bladder: report of 5 cases with immunohistologic study. Urology. 1994; 43:389-93.

7. Perret L, Chaubert P, Hessler D, Guillou L. Primary heterologous carcinosarcoma (metaplastic carcinoma) of the urinary bladder: a clinicopathologic, immunohistochemical and ultrastructural analysis of eight cases and a review of the literature. Cancer. 1998; 82:1535-49.

8. Wang J, Wang FW, Lagrange C, et al. Clinical features of sarcomatoid carcinoma (carcinosarcoma) of the urinary bladder: analysis of 221 cases. Sarcoma. 2010; 2010:454-792.

9. Atilgan D, Gencten Y. Carcinosarcoma of the bladder: a case 
report and review of the literature. Case Rep Urol. 2013; 2013:716704.

10. Sreenan JJ, Hart WR. Carcinosarcomas of the female genital tract. A pathologic study of 29 metastatic tumors: Further evidence for the dominant role of the epithelial component and the conversion theory of histogenesis. Am J Surg Pathol. 1995; 19:666-74.

11. Wright JL, Black PC, Brown GA, et al. Differences in survival among patients with sarcomatoid carcinoma and urothelial carcinoma of the bladder. J Urol. 2007; 178:2302-07.

12. Rogers CG, Palapattu GS, Shariat SF, et al. Clinical outcomes following radical cystectomy for primary nontransitional cell carcinoma of the bladder compared to transitional cell carcinoma of the bladder. J Urol. 2006; 175:2048-53.

\section{Correspondence}

Ismail Basibuyuk, MD

dr.ismailbb@gmail.com

Cizre State Hospital, Department of Urology, Sirnak, Turkey

Ramazan Topaktaş, MD (Corresponding Author)

ramazantopaktas@yahoo.com

Haydarpasa Numune Training and Research Hospital,

Department of Urology

Tibbiye street Number:23, 34668 Istanbul, Turkey

Fatih Elbir, MD

drfatihelbir@gmail.com Mardin State Hospital, Department of Urology,

Mardin, Turkey 